\title{
VOTO ESTRUCTURAL, RACIONAL Y MEDIATIZADO EN LAS ELECCIONES GENERALES ESPAÑOLAS DE 1996, $2000 \mathrm{Y}$ 2004
}

\author{
STRUCTURAL, RATIONAL, AND MEDIATIZED VOTE IN THE SPANISH \\ GENERAL ELECTIONS FROM 1996, 2000 AND 2004
}

\author{
JUAN JesÚs GONZÁLEZ \\ Universidad Nacional de Educación a Distancia (UNED). España \\ jgonzalez@poli.uned.es
}

\begin{abstract}
RESUMEN
El artículo presenta tres modelos de voto que pretenden explicar aquello que cada una de las tres últimas elecciones generales celebradas en España tiene de específico. Así, se propone un modelo estructural para las elecciones de 1996, un modelo racional-económico para las elecciones de 2000 y un modelo mediatizado para explicar los mecanismos que intervinieron en las elecciones de 2004. El artículo presenta por último un modelo de voto que pretende integrar las principales conclusiones extraídas de cada uno de los modelos anteriores. Se hace especial énfasis en los mecanismos comunicativos de las últimas elecciones (2004), subrayando la influencia de los medios sobre la atribución de responsabilidad en virtud de la cual los votantes evalúan las políticas públicas, al tiempo que se llama la atención sobre el papel activo de las audiencias a la hora de seleccionar aquellos mensajes que son coherentes con su experiencia personal y con su patrón cultural.
\end{abstract}

\section{Palabras Clave Adicionales}

Anclaje electoral, Guerra de encuadres, Modelos de voto, Sistema mediático, Voto económico.

\begin{abstract}
The article presents three voting models that attempt to explain what is specific about each of the last three general elections held in Spain. Thus, a structural model is proposed for the 1996 elections, a rational-economic model for the elections in 2000, and a mediatized model to explain the mechanisms that intervened in the 2004 elections. The article then presents a voting model that attempts to integrate the main conclusions drawn from each of the previous models. Special emphasis is placed on the communicational mechanisms of the last elections (2004), underlining the influence of the media in attributing responsibility, the issue according to which voters evaluate public policy. Simultaneously, attention is called to the active role of the audiences in selecting the messages that are coherent with their personal experience and their cultural pattern.
\end{abstract}

\section{Additional KeYWORdS}

Economic Voting, Electoral Cleavage, Framing War, Media System, Voting Models. 


\section{INTRODUCCIÓN ${ }^{1}$. HACIA UNA DEMOCRACIA CENTRADA EN LOS MEDIOS}

En este artículo propongo tres modelos de voto con los que trato de explicar las tres últimas elecciones generales celebradas en España. Comenzaré presentado un modelo estructural con el que intento explicar lo ocurrido en las elecciones generales de 1996. A continuación, presentaré un modelo de voto económico que toma como referencia las elecciones generales de 2000 y, por último, presentaré un modelo de voto que denomino mediatizado y que intenta explicar el desenlace final de las elecciones generales de 2004, celebradas tras los atentados del 11-M. Cada uno de los modelos pretende explicar lo que considero distintivo o característico de las tres elecciones que tomo como referencia. De hecho, cada una de estas elecciones plantea una pregunta de interés. Así, por ejemplo, las elecciones generales de 1996 nos plantean la pregunta por las condiciones que hicieron posible el cambio y la alternancia dentro de una pauta de gran estabilidad electoral, es decir sin que se produjese el vuelco electoral que anticipaban las encuestas (González, 1998). En segundo lugar, las elecciones de 2000 nos plantean la pregunta por las condiciones que hicieron posible que un líder como José María Aznar, que aparecía en los sondeos muy escorado a la derecha en términos de la escala ideológica, consiguiese una victoria por mayoría absoluta. Por último, las elecciones de 2004 nos plantean la pregunta por las condiciones que hicieron posible que el Partido Socialista Obrero Español (PSOE) ganase por 5 puntos de diferencia, invirtiendo a su favor la distancia que la encuesta preelectoral del Centro de Investigaciones Sociológicas (CIS) daba tan sólo un mes antes de las elecciones (que era de 5 puntos a favor del Partido Popular (PP)). Pues bien, mi propósito es presentar un modelo de voto estructural para responder a la primera pregunta, un modelo de voto racional-económico para responder a la segunda y un modelo de voto mediatizado para responder a la tercera. Dado que las dos primeras elecciones han sido estudiadas en artículos anteriores (González, 1996 y 2002), me detendré en particular en las últimas elecciones y en la exposición del modelo mediatizado.

Cabría distinguir, desde esta perspectiva, entre el plano estructural, el plano de evaluación racional y el plano de la opinión pública. La importancia relativa de estos planos varía de unas elecciones a otras, pero se trata de planos, a un mismo tiempo, autónomos e interrelacionados, de tal suerte que, si bien cada uno de ellos tiene una lógica distinta, los tres modelos propuestos pueden llegar a ser complementarios, permitiendo un uso integrado y conjunto. Propongo, en consecuencia, un modelo de voto integrado, en el que distingo dos tipos de variable: variables de control y variables explicativas. Quiero decir con esto que los factores que intervienen en el proceso de decisión electoral pueden hacerlo de dos maneras: $\mathrm{o}$ bien de manera directa o bien a través de otras variables. En el primer caso, hablaré de variables explicativas, por cuanto ejercen una influencia directa, en tanto que, en el segundo caso, hablaré de variables de control, por cuanto su influencia

${ }^{1}$ El autor agradece las sugerencias y comentarios de tres evaluaciones anónimas. 
se ejerce a través de las primeras. Así, por ejemplo, considero a los factores estructurales como la clase social o la edad variables de control, y considero la experiencia personal o la cultura política variables explicativas propiamente dichas, ya que la clase social o la edad operan a través de éstas u otras variables. No obstante, la principal variable explicativa es la influencia de los medios, no sólo porque los medios sean capaces de imponer a las audiencias su agenda temática, sino porque cada vez tienen más capacidad para conformar la atribución de responsabilidad y de influir, a través de ella, sobre la evaluación de las políticas públicas, la cual constituye el eje central del modelo que propongo como conclusión. Este modelo final es, al mismo tiempo, sintético, por cuanto integra los modelos previos, y explicativo, por cuanto propone la siguiente secuencia causal: agenda de los medios [priming/framing] - atribución de responsabilidad - evaluación de políticas (lyengar, 1991; Entman, 1993; McCombs; 2004).

A tal fin, comenzaré presentando un modelo estructural para dar cuenta de lo que las elecciones de 1996 tienen, a mi juicio, de específico: la mencionada combinación de estabilidad y cambio 0 , dicho con otras palabras, de cambio y alternancia dentro de una pauta de relativa estabilidad electoral. La hipótesis en que me apoyo tiene sus antecedentes en la literatura sobre las bases sociales de la democracia (Lipset y Rokkan, 1967 [1992]; Przeworski, 1985 [1988]; Przeworski y Sprague, 1986; Esping-Andersen, 1985, 1990 [1993]) y parte de la distinción entre factores de estabilidad y factores de cambio. En este caso, presentaré un estudio del perfil sociodemográfico de las bases de los partidos, comparando las elecciones de 1986, 1996 y 2004, para mostrar que el factor de estabilidad ha sido la clase social, en tanto que el factor de cambio ha sido la edad.

A continuación, presentaré un modelo de voto económico para dar cuenta de las elecciones de 2000 y de la victoria del PP por mayoría absoluta. Recurriré a la perspectiva micro, a fin de mostrar que la evaluación de políticas públicas se ha convertido en un momento crucial de la decisión de voto (González, 2002). El modelo se inspira en abundante literatura sobre voto económico (Lewis-Beck y Paldam, 2000), si bien permite diferenciar dos componentes que aparecen frecuentemente confundidos en la discusión sobre voto económico: el componente que llamo factor bonanza, asociado a la percepción de la situación económica, y el factor competencia, asociado a la percepción de la capacidad de los partidos para gestionar la economía.

Por último, adoptaré una perspectiva macro-micro para dar cuenta del vuelco electoral registrado en las elecciones de 2004. Presentaré, por un lado, las estrategias de la prensa de referencia para priorizar (priming) y encuadrar (framing) la campaña electoral, deteniéndome en particular en el conflicto de encuadres que se produce a partir de los atentados terroristas del 11-M (Olmeda, 2005 y Sampedro, 2005). Me detendré, por otro lado, en el estudio de la percepción de dicho conflicto por parte de un grupo de votantes de centro, así como de los esquemas de voto con que estos votantes respondieron a la crisis producida por los atentados. En este caso, la hipótesis explicativa radica en la capacidad de los medios no sólo para imponer a las audiencias su agenda temática, sino también para conformar la atribución de responsabilidad y de influir, a través de ella, en la evaluación de las políticas públicas. 
Esta secuencia analítica pretende llamar la atención sobre una tendencia de la democracia, en general, y de la española, en particular, hacia lo que se ha dado en llamar democracia de audiencia y que, en nuestro caso, sería más correcto caracterizar como una democracia centrada en los medios (Swanson, 1995), por cuanto el protagonismo cada vez más indiscutible de los medios no implica, por desgracia, la existencia de una esfera pública al servicio de un electorado juicioso y bien informado (tal como presupone la democracia de audiencia: Manin, 1998), sino un sistema mediático polarizado que, lejos de arbitrar y regular el conflicto político, se convierte en parte activa de dicho conflicto.

\section{LAS ELECCIONES GeNERALES DE 1996. EL PESO DE LOS FACTORES ESTRUCTURALES}

Antes de presentar los modelos en cuestión, voy a establecer el marco teórico-analítico de cada uno de ellos. El marco teórico del modelo estructural remite, en último término, al estudio clásico de Lipset y Rokkan sobre los sistemas de partido y los alineamientos electorales (1967) [1992]. En él se aborda la cuestión de cómo las divisiones sociales tradicionales por razón de clase social, lengua o religión se traducen en la configuración de determinados sistemas de partido. Más concretamente, estos autores analizan el impacto de las dos grandes revoluciones del mundo occidental, la revolución nacional y la industrial, sobre dicha configuración. Desde esta perspectiva, la primera de ellas (la revolución nacional) ha dado lugar a conflictos en el eje centro-periferia ("el conflicto entre la cultura central que construye la nación y la resistencia creciente de las poblaciones sometidas de las provincias y las periferias, étnica, lingüística o religiosamente diferenciadas" (Ibíd.: 245). En tanto que la segunda ha dado lugar a conflictos de clase que fueron especialmente virulentos en la primera mitad del siglo XX, pero que, como señalaban Lipset y Rokkan en los años sesenta, se habían suavizado con posterioridad a la segunda guerra mundial, debido a una diversidad de factores: "la experiencia de cooperación nacional durante la guerra, las mejoras del nivel de vida en los años cincuenta, el rápido crecimiento de una nueva clase media...". Con todo, el factor más importante era, a juicio de estos autores, "el asentamiento de los partidos obreros en estructuras de gobierno locales y nacionales, y su consiguiente domesticación dentro del sistema" (1967: 254-255).

Al examinar el caso español, podemos comprobar, en efecto, que el sistema de partidos de la transición se configuró con arreglo a estos dos ejes: el eje centro-periferia que contrapone partidos de ámbito nacional español a los nacionalismos periféricos de base étnico-cultural (Giner y Moreno, 1990) y el eje izquierda-derecha, al que me voy a referir principalmente. Este segundo eje ha mostrado una gran estabilidad a lo largo de estas casi tres décadas de experiencia democrática, que ha sido posible en virtud de una serie de anclajes estructurales (cleavages) que han servido de apoyo a la contraposición izquierda-derecha y, con ello, a las ideologías de uno u otro signo. A lo largo de esta experiencia, la clase social ha jugado un papel relevante como factor de estabilidad, si bien 
en ocasiones ha perdido influencia a favor de otros factores. Desde este punto de vista, podemos distinguir entre factores que han dado estabilidad al proceso electoral, como la clase, y factores de cambio, como la edad, tal como veremos más adelante.

Esta línea de análisis ha tropezado con dos tipos de problema: uno de orden empírico y otro de orden teórico. El primero de ellos se deriva de la presunción, postulada por el citado Lipset (Lipset et al., 1991 y 1993), de que la pérdida de centralidad de la clase obrera dejaba al análisis de clase fatalmente destinado a la inoperancia. Tal como he defendido en otra ocasión, una cosa es dicha pérdida de centralidad, fácilmente identificable desde un punto de vista empírico, y otra bien distinta que el análisis de clase se acabe con ella (González, 1996). El segundo problema radica en formular teóricamente la relación entre posición de clase y preferencias electorales. En este punto, las teorías pluralistas representadas por autores como el recientemente desaparecido Lipset tendían a dar por supuesto que la primera determinaba las segundas, lo que permitía llegar fácilmente a la conclusión de que, con el advenimiento de la sociedad pos-industrial y la pérdida de protagonismo de la clase obrera, los partidos de izquierda perdían su razón de ser.

En este punto, varios autores han introducido la política como variable fundamental para entender la relación entre clase y voto. El punto de partida no es otro que la distinción entre estructura y formación de clase, donde la política aparece como variable que vincula la posición de los individuos en la estructura social con su conformación como actores políticos. En el caso de autores como Przeworski (Przeworski, 1985 [1988]; Przeworski y Sprague, 1986), la evolución electoral de los partidos socialdemócratas es una consecuencia de su propia movilización política. En el caso de Esping-Andersen (1985, 1990 [1993]), la clave de su éxito radica en su capacidad para construir coaliciones interclasistas.

Por mi parte, he aplicado esta perspectiva analítica al caso español con resultados que he tratado de resumir en González (2005), donde he subrayado el papel de las políticas sociales. En este punto me aparto de autores como Maravall (2003) que han interpretado las políticas de la etapa socialista como parte de una estrategia de compensación en virtud de la cual el gobierno socialista habría utilizado las políticas sociales para compensar los costes de las políticas económicas de ajuste. Por el contrario, las políticas sociales de la etapa socialista, y especialmente el esfuerzo redistributivo de su segunda mitad, se entienden mejor como resultado del conflicto entre los sindicatos y el gobierno, toda vez que el desenlace de la huelga general de 1988 permitió a los primeros imponer sus reivindicaciones sobre el segundo. Esto tuvo dos efectos aparentemente contradictorios pero que, desde mi perspectiva, no lo son: a corto plazo, mantuvo estables los alineamientos político-electorales mientras el principal partido de la oposición, el PP, amagaba con políticas de corte neoliberal, tal como ocurrió en las elecciones de 1993 y 1996. Por otro, facilitó el trasvase de voto obrero al PP una vez que éste llegó al gobierno y renunció a políticas de este tipo, que fueron sustituidas por la concertación y el diálogo social. De ahí que el debilitamiento del voto de clase no se produjese en 1996, sino en 2000 (Caínzos, 2001: 160 y ss.).

Ahora bien, si la continuidad de los alineamientos electorales y en particular los de corte clasista explican la estabilidad electoral de 1996, la edad explica el cambio. La 
razón es simple: el esfuerzo redistributivo de la segunda mitad de la etapa socialista no fue tanto a favor de las clases trabajadoras como a favor de las clases pasivas, es decir fue una redistribución a favor de los viejos y en contra de los jóvenes. De ahí que estos, que habían sido uno de los baluartes del gobierno socialista en sus primeros tiempos, se volvieran contra él.

A fin de apoyar empíricamente lo que acabo de exponer, voy a presentar un sencillo modelo de regresión logística que toma como variable dependiente el voto PP en oposición al PSOE y que toma como variables independientes tres variables de corte estructural: la clase social, la edad y el hábitat. He aplicado el modelo a tres elecciones separadas entre sí por un periodo de ocho o diez años, lo que arroja un periodo de observación que va de las elecciones de 1986 a las de 2004 (tabla 1). Tal como adelanté, el objetivo es explicar el rasgo distintivo de las elecciones de 1996: la combinación de estabilidad y cambio. Pues bien, del modelo se desprende que mientras los alineamientos de clase se mantuvieron estables a lo largo del periodo, el coeficiente de la edad cambió de signo en las elecciones de 1996, lo que quiere decir que los jóvenes se movilizaron contra el partido socialista para echarlo del gobierno.

Tabla 1.

Modelo de regresión logística para estimar la influencia de los factores estructurales (1986-2004).

Variable dependiente: voto PSOE (1) frente a voto PP (0).

\begin{tabular}{|c|c|c|c|}
\hline & $\begin{array}{c}1986 \\
\text { Coef. (error) Signif. }\end{array}$ & $\begin{array}{c}1996 \\
\text { Coef. (error) Signif. }\end{array}$ & $\begin{array}{c}2004 \\
\text { Coef. (error) Signif. }\end{array}$ \\
\hline \multicolumn{4}{|c|}{ Clase (ref.: Autónomo) } \\
\hline Profesional &,$- 09(0,08)$ &,$- 15(0,10)$ &,$- 02(0,14)$ \\
\hline No manual & $0,15(0,07)^{*}$ & $0,26(0,09)^{\star *}$ & $0,30(0,14)^{*}$ \\
\hline Manual cualificado & $0,86(0,07)^{\star *}$ & $1,17(0,09)^{\star *}$ & $0,91(0,13)^{\star *}$ \\
\hline Manual no cualif. & $1,10(0,07)^{\star *}$ & $1,09(0,08)^{\star *}$ & $0,90(0,13)^{* *}$ \\
\hline \multicolumn{4}{|l|}{ Edad (ref.: >54) } \\
\hline$<35$ & $0,60(0,05)^{* *}$ &,$- 17(0,07)^{\star *}$ & $0,78(0,10)^{* *}$ \\
\hline $35-54$ & $0,08(0,05)$ &,$- 03(0,07)$ & $0,43(0,10)^{* *}$ \\
\hline \multicolumn{4}{|l|}{ Hábitat (ref.: <10 mil) } \\
\hline $10-100 \mathrm{mil}$ & $0,52(0,05)^{* *}$ & $0,03(0,07)$ & $0,26(0,11)^{* *}$ \\
\hline$>100 \mathrm{mil}$ & $0,53(0,05)^{* *}$ &,$- 04(0,07)$ & $0,13(0,11)$ \\
\hline N. ${ }^{\circ}$ de casos & 10716 & 5594 & 2700 \\
\hline Chi cuadrado & 868,8 & 425,4 & 174,8 \\
\hline Pseudo R2 & 0,11 & 0,10 & 0,08 \\
\hline$\%$ casos correctos & $63,5 \%$ & $63,3 \%$ & $61,8 \%$ \\
\hline
\end{tabular}


En suma, el estudio de las elecciones de 1996 recomienda prestar atención a los factores estructurales, que constituyen el primer paso en la construcción de nuestro "modelo integrado de voto" (véase la parte superior de la figura que presento al final). Llamaremos a este primer tipo de factores variables de control, para distinguirlas de las variables explicativas propiamente dichas. Ya he adelantado la razón de esta distinción: mientras las variables explicativas ejercen una influencia directa sobre la decisión de voto, las variables de control ejercen influencia a través de otras variables. Más adelante volveré sobre este punto. Baste con decir, por el momento, que con el estudio de las elecciones de 2000, al que pasamos a continuación, comenzamos a identificar algunas de estas variables explicativas.

\section{LAS ELECCIONES GENERALES DE 2000: EL VOTO ECONÓMICO}

No hace falta recordar que la situación política de las elecciones de 2000 fue muy distinta de la de 1996. Quizá la principal diferencia fue que así como las elecciones de 1996 estuvieron muy condicionadas por la incertidumbre sobre la estrategia a seguir por parte del PP, el desarrollo de la legislatura 1996-2000 había despejado muchas incógnitas a este respecto. Por poner dos ejemplos sobresalientes, el PP pasó de un discurso antinacionalista a coaligarse con los partidos de este signo, al tiempo que pasó de las proclamas neoliberales a un diálogo social sin precedentes.

En consecuencia, la apurada victoria popular en las elecciones de 1996 propició un giro estratégico del PP que dio como resultado una combinación de bonanza económica y paz social. No es cosa de discutir aquí sobre la génesis y el desarrollo de esta experiencia (González, 2002), sino tan sólo recordar algunos presupuestos teóricos, entre los que hay que destacar el estudio de Morris Fiorina sobre el voto retrospectivo en las elecciones presidenciales estadounidenses (1981), que sirvió para establecer el principio general de que los votantes tienden a hacer responsables a los gobiernos por la gestión de la economía. A partir de ahí, se produjo un auténtico aluvión de literatura sobre voto económico que, a la altura del año 2000 (coincidiendo con nuestras elecciones de referencia), arrojaba un saldo de más de 200 contribuciones relevantes. Según un número monográfico de la revista Electoral Studies aparecido ese mismo año 2000 (Lewis-Beck y Paldam, 2000), los principales hallazgos de dicha literatura se podían formular en los siguientes términos:

- El componente económico del voto daba cuenta de aproximadamente un tercio de las variaciones totales observables de voto.

- Las principales variables influyentes eran la tasa de crecimiento de la economía, el desempleo y la inflación.

- El componente retrospectivo del voto tenía más influencia que el componente prospectivo. 
- El componente sociotrópico (las condiciones generales del país) tenía, a su vez, más influencia que el egotrópico (la situación personal o familiar).

- Los votantes son más sensibles al corto que al largo plazo.

Estos supuestos contribuyeron a asentar el modelo restringido de voto económico, en virtud del cual los votantes premian o castigan al partido en el gobierno por la gestión económica realizada. Este modelo inicial se fue complicando y enriqueciendo poco a poco hasta llegar, en el caso español, al modelo de voto económico ampliado, tal como aparece en una serie de estudios entre los que destaca el de Marta Fraile sobre la etapa socialista (Fraile, 2005). De acuerdo con este estudio, el protagonismo de la oferta política ha estado muy repartido entre las políticas económicas y las políticas de bienestar, hasta el punto de que las políticas sociales jugaron un papel decisivo en la permanencia de los socialistas en el gobierno. Más concretamente, el estudio demuestra que la desconfianza de los electores acerca de cuáles serían las políticas sociales del PP fue crucial en las elecciones de 1993 y 1996, de lo que se deduce que si el PP hubiera anticipado en los primeros años noventa las políticas sociales que terminó haciendo una vez que llegó al gobierno (en lugar de amagar con proclamas neoliberales), la etapa socialista hubiera sido seguramente más corta.

En suma, el modelo ampliado no hace sino integrar una serie de políticas que el modelo original no contemplaba, debido a la irrelevancia de las políticas sociales en el caso estadounidense. Otros autores, como la citada Marta Fraile, se han encargado de aplicar este modelo a las elecciones de 2000 (Fraile, 2002), así que lo que voy a presentar es un modelo sencillo de voto económico restringido. Ahora bien, antes de presentar este modelo me gustaría recordar el rasgo distintivo de las elecciones de 2000, a fin de responder a la pregunta que plantean estas elecciones: ¿cómo explicar que un líder como Aznar, que estuvo siempre muy escorado a la derecha en términos de la escala ideológica, consiguiese una victoria por mayoría absoluta? Pues bien, la respuesta es que el PP supo aprovechar una coyuntura de máxima disonancia entre los factores de evaluación racional y el componente ideológico del voto, siendo esta disonancia el rasgo distintivo de aquellas elecciones (González, 2002). De acuerdo con este análisis, la posición del votante medio se caracterizaba por auto ubicarse un punto más cerca del PSOE que del PP, al tiempo que calificaba la capacidad del PP para gestionar las diez políticas públicas en cuestión ${ }^{2}$ un punto mejor que la del PSOE (once décimas, para ser exactos). Esta tensión entre una mayor proximidad ideológica al PSOE y una mejor evaluación racional del PP constituye la clave de aquellas elecciones, saldadas a favor de la evaluación racional (González, 2002: 24).

Con esta premisa, presento un modelo estadístico de voto económico restringido que intenta dar cuenta, en el marco de este predominio del voto racional, del efecto específico

\footnotetext{
${ }^{2}$ Economía, empleo, impuestos, educación, sanidad, vivienda, inmigración, política autonómica, política europea y medio ambiente.
} 
de los factores económicos. Estos factores pueden ser de dos tipos: los relativos a la situación económica, que llamo factor de bonanza, y los relativos a la confianza en la capacidad de gestión de los partidos, que llamo factor de competencia. A fin de mostrar la existencia de este doble componente de voto económico en las elecciones de 2000 , el efecto de la valoración de la capacidad de gestión de los partidos sobre la decisión de voto está controlado por el efecto de la bonanza económica o, en otras palabras, por la percepción de la situación económica ${ }^{3}$.

La interpretación del modelo que aparece en la tabla 2 es muy sencilla: controlando por clase social y distancia ideológica, el efecto neto del factor bonanza fue muy importante (primera columna de la tabla 1), tal como corresponde a una coyuntura económica muy positiva, pero si introducimos en el modelo el factor competencia (segunda columna de la tabla 2), podemos observar que el efecto neto de este segundo factor fue casi tan importante como el primero, como corresponde a una mayor confianza en la capacidad de gestión del gobierno en comparación con la oposición, lo que recomienda diferenciar ambos componentes.

Resumiendo este segundo modelo, las elecciones de 2000 sugieren que la evaluación racional de las políticas públicas ocupa un lugar central en la explicación del voto, no sólo por contraste con la ideología (que juega un papel de variable de control en el modelo que propongo), sino también por contraste con factores que podemos llamar de experiencia personal, tales como la percepción de una determinada situación económica. Pues la clave del voto de 2000 no reside tanto en la percepción de una situación económica marcada por la bonanza como en la atribución de responsabilidad de dicha bonanza a la capacidad de los partidos para la buena gestión de la economía. A continuación, aprovecharemos las elecciones de 2004 para adentrarnos en los mecanismos de atribución de responsabilidad y para estudiar el papel de los medios de comunicación en dicha atribución.

\section{LAS ELECCIONES GENERALES DE 2004: LA EMERGENCIA DEL VOTO MEDIATIZADO}

\section{Marco analítico}

Llegamos así a las elecciones de 2004, para las cuales vamos a proponer un modelo de voto que llamo mediatizado. Tal como adelantamos, la pregunta que nos plantean estas elecciones es la de los mecanismos en virtud de los cuales el PSOE fue capaz de dar la

\footnotetext{
${ }^{3}$ La variable dependiente del modelo es dicotómica: voto PP (1) versus voto a PSOE o IU (0). Las variables de control son la clase social (modelo Goldthorpe de cinco categorías; González, 1992) y la distancia ideológica, estimada como la diferencia de las distancias respecto a los dos principales partidos: PP y PSOE. Por lo que se refiere a las variables independientes, el factor bonanza está elaborado a partir de dos preguntas, una sobre la valoración de la situación económica general de España y otra sobre la situación económica familiar.
} 
Tabla 2.

Modelo de regresión logística para estimar el doble componente económico del voto (factor bonanza y factor competencia).

Variable dependiente: voto PP (1) frente a voto PSOE o IU (0).

\begin{tabular}{lcr}
\hline & $\begin{array}{c}\text { Factor bonanza } \\
\text { Coeficiente (error) Signif. }\end{array}$ & $\begin{array}{r}\text { Factor competencia } \\
\text { Coeficiente (error) Signif. }\end{array}$ \\
\hline Clase (Cat. Ref.: manual cualificado) & $1,13(0,27)^{* *}$ & $1,17(0,28)^{* *}$ \\
Autónomo & $0,54(0,23)^{*}$ & $0,40(0,24)$ \\
Profesional & $0,84(0,21)^{* *}$ & $0,72(0,22)^{* *}$ \\
No manual & $0,47(0,20)^{*}$ & $0,41(0,21)^{*}$ \\
Manual no cualificado & & \\
Bonanza (Cat. Ref.: doble valoración & & $1,53(0,19)^{* *}$ \\
negativa) & $1,71(0,18)^{* *}$ & $1,19(0,21)^{* *}$ \\
Todo positivo & $1,34(0,20)^{* *}$ & $0,08(0,25)$ \\
General positivo & $0,07(0,24)$ & \\
Particular positivo & - & $1,42(0,17)^{* *}$ \\
Competencia (Cat. Ref.: otra respuesta) & - & $-0,72(0,25)^{* *}$ \\
PP más capacitado & - & 1609 \\
PSOE más capacitado & 1609 & 1066,7 \\
Distancia ideológica (variable continua) & $0,58(0,03)^{* *}$ & 0,49 \\
N. ${ }^{*}$ de casos & 976,8 & $84,8 \%$ \\
Chi cuadrado & 0,46 & $(0,03)^{* *}$ \\
Pseudo R2 & $83,7 \%$ & \\
\% casos correctos & & \\
\hline
\end{tabular}

Fuente: CIS, estudio panel 2382-4, febrero-marzo 2000. * Significación al 95\%. * * Significación al 99\%.

vuelta a un pronóstico adverso: desventaja de 5 puntos porcentuales, según la encuesta preelectoral del CIS. Parece claro, en principio, que dichos mecanismos fueron de naturaleza eminentemente comunicativa y que tienen que ver con la batalla mediática que se libró tras los atentados del 11-M.

En este punto, nos encontramos con un problema, por cuanto la encuesta postelectoral del CIS de 2004 permite estimar la magnitud de la movilización electoral producida por el 11-M, pero no sus mecanismos. La estimación de la movilización es la siguiente:

Por último, el factor competencia se compone de tres categorías: si el PSOE hubiera estado en el gobierno en los últimos cuatro años la economía estaría peor ("PP más capacitado" en la tabla), la contraria ("PSOE más capacitado") y otra respuesta (categoría de referencia). 
a) EI PSOE movilizó entre mediados de febrero (fecha de la encuesta preelectoral del $\mathrm{CIS}$ ) y el día de las elecciones en torno a dos millones de electores.

b) En su mayor parte (del orden de un millón setecientos mil) se movilizaron a raíz del 11-M.

c) Un millón manifestó que la principal razón de su voto fue el 11-M.

Se trata, por tanto, de una movilización electoral sin precedentes que, según todos los indicios, se produjo durante el fin de semana. Ahora bien, el enigma del 11-M radica en que la encuesta postelectoral del CIS no permite explicar los mecanismos concretos del vuelco electoral, más allá de la cuantificación de su impacto movilizador. Queda la duda, por tanto, sobre los mecanismos de la decisión electoral, que es el objeto de este tercer modelo, en el que presento la percepción y la reacción de un grupo estratégico de votantes ante el conflicto mediático que siguió al 11-M, para lo cual procederé de la forma siguiente: comenzaré por caracterizar el sistema de los medios de comunicación (media system) en España, deteniéndome, en particular, en los mecanismos que utilizan para influir sobre la opinión pública. A continuación describiré los alineamientos y las estrategias de los medios durante la (pre)campaña electoral de 2004 y terminaré presentando el conflicto mediático del 11-M con ayuda de entrevistas postelectorales. No es mi intención, por tanto, presentar el conflicto comunicativo entre el gobierno y los medios de comunicación que se opusieron a él (eso ya lo han hecho autores como Olmeda, 2005 y Sampedro, 2005), sino identificar la percepción del mismo por parte de aquellos votantes que estaban llamados a ser árbitros de las elecciones, en tanto que votantes de centro que tenían ya experiencia de votar tanto al PSOE como al PP.

Parece claro que el rasgo distintivo de las elecciones generales de 2004 y lo que las aparta de cualquier otra celebrada hasta entonces fue el protagonismo de los medios de comunicación. Hablar de la relación entre política y medios en nuestro días se ha convertido, más que en un tópico, en una redundancia, por cuanto ambas cosas han llegado a ser inseparables, hasta el punto de que algunos autores se refieren a las sociedades postindustriales como sociedades mediatizadas, entendiendo por tales "sociedades en que las prácticas sociales [...] se transforman por el hecho de que hay medios" (Verón 1998: 124). Por lo que se refiere concretamente a la política, la presencia de los medios ha contribuido a hacer más transparente el proceso democrático, pero los medios han llegado a colonizar la relación entre partidos y ciudadanos hasta el punto de que ya no está claro, en ocasiones, si la política que perciben los ciudadanos se corresponde con la oferta de los partidos o es simplemente un producto de los medios y de su omnipresencia (Meyer, 2002). Hay momentos, sin embargo, en que la actuación de los medios es irreemplazable, tal como ocurre durante las campañas electorales, y es en esos momentos cuando la política se convierte en política mediatizada por definición, entendiendo por tal una política concebida por y para los medios. Pues no es sólo que las campañas se hagan pensando en los medios, más que en los votantes, sino que convierten la política en un espectáculo mediático equiparable a cualquier otra modalidad de "info-tenimiento". 
Así pues, entender la dinámica de las elecciones de 2004 pasa, por lo pronto, por entender el funcionamiento del sistema de medios en España. A la hora de caracterizar este sistema, podemos tomar como referencia el estudio de Hallin y Mancini (2004) que clasifica los media systems según tres grandes tipos: el modelo liberal (anglosajón), el modelo "corporativo democrático" del centro y norte de Europa y el modelo mediterráneo de "pluralismo polarizado". La clasificación atiende a cuatro variables o dimensiones principales: la circulación de prensa, el nivel de profesionalización de los periodistas, la politización de los medios y el grado de intervención estatal sobre los mismos (Ibíd.: 21 ss). A efectos de nuestro argumento, conviene subrayar que Hallin y Mancini incluyen a España en el modelo mediterráneo debido, entre otras razones, al alineamiento político de los medios (Ibíd.: 106 ss). En último término, este alineamiento político de los medios les apartaría de su primigenia misión informativa y les empujaría hacia tareas de adoctrinamiento propias de la prensa de partido de otra época o de un aparato ideológico. En consecuencia, los medios proporcionan una representación de la escena política que dramatiza las diferencias y antagonismos entre unas opciones y otras, dando apariencia de confrontación donde a menudo hay convergencia, a fin de atraer la atención de las audiencias. Asimismo, los medios buscan noticias caracterizadas no tanto por su relevancia como por su espectacularidad, con el riesgo consiguiente de deslizamiento hacia lo que Castells ha llamado la "política del escándalo" (2003: 426 y ss.).

Con estas premisas, la primera tarea de los medios no es otra que la elaboración de un temario 0 , si se quiere, de una agenda temática destinada a fijar el foco de atención de la audiencia (McCombs, 2004). No se trata, por el momento, de sugerir a la audiencia código alguno de interpretación de los mensajes, sino tan sólo de establecer el sendero temático por el que la audiencia debe circular (agenda setting). El éxito de esta primera tarea depende de la capacidad de los medios para que sus audiencias interioricen la agenda temática y la hagan suya. En la medida en que la agenda temática de los medios y la agenda personal de sus audiencias confluyen, haciendo que las preocupaciones de los ciudadanos se identifiquen con aquélla, los medios habrán conseguido su primer objetivo. No se trata de un objetivo fácil, toda vez que el conocimiento de los ciudadanos se nutre de otras muchas fuentes, empezando por su propia experiencia en el entorno familiar, social y laboral, lo que requiere de una cierta correspondencia entre la agenda temática de los medios y los problemas cotidianos de los ciudadanos.

La segunda tarea de los medios es el encuadramiento de los mensajes, entendiendo por tal la "selección de algunos aspectos de la realidad haciéndolos más relevantes, a fin de promover una particular definición del problema, una interpretación de sus causas, una evaluación moral y/o una recomendación acerca del tratamiento del asunto en cuestión" (Entman, 1993: 52). El encuadramiento puede ser de dos tipos: episódico o temático. Mientras el primero proporciona imágenes o descripciones más o menos espectaculares pero relatadas en términos episódicos referidos a sucesos particulares y concretos, el encuadramiento temático proporciona una interpretación más general sobre la causalidad del problema que sirve de trasfondo a la noticia. Esta diferencia es fundamental, por cuanto está asociada a diferentes mecanismos de atribución de responsabilidad por parte de la 
audiencia (lyengar, 1991) .

Otro mecanismo bien conocido de la comunicación es el priming, que significa dos cosas: primar o imprimar. Esta doble acepción del priming permite a los medios, por un lado, primar o priorizar determinados temas que llevan asociados atributos de fortaleza 0 debilidad respecto a un actor político; así, por ejemplo, el diario El País puede centrar el foco de atención de una campaña electoral en noticias relacionadas con políticas sociales a sabiendas de que éste es un punto fuerte de los socialistas frente al PP. En tanto que, por otro lado, priming significa imprimar, a la manera de un lienzo, la presentación de un tema por su asociación con actitudes o predisposiciones más o menos latentes entre los electores. En otras palabras, imprimar significa emitir un mensaje con el fin de activar ideas o pensamientos conectados con él (Entman, 2004: 27).

Es fácil encontrar ejemplos de priming en el caso que nos ocupa (la campaña de 2004). De un lado, El País recordó durante la campaña las afirmaciones de Aznar sobre las (presuntas) armas de destrucción masiva que sirvieron de coartada para la invasión de Irak, lo que sirvió de priming para la posterior denuncia de manipulación por la gestión del 11-M, como enseguida veremos. Por su parte, las cadenas públicas controladas por el PP (Televisión Española -TVE- y Telemadrid) emitieron el documental "Asesinato en febrero" (sobre el asesinato de Fernando Buesa poco antes de las elecciones de 2000) tras el 11-M, a fin de reforzar el argumento del gobierno sobre la autoría de ETA.

En suma, encuadrar las noticias (framing), priorizar los temas (primera acepción del priming) e imprimar la percepción de las audiencias a fin de poner en relación los mensajes con sentimientos e ideas preexistentes (recordemos que priming también significa cebo en su segunda acepción) son las tareas principales de los medios en la comunicación política de nuestros días. Aunque es habitual entre los analistas prevenir al lector sobre el potencial de manipulación que todo ello entraña cuando la comunicación va dirigida a audiencias poco informadas, puede que sea el momento de recordar que, debido a los costes de información que plantea el seguimiento de la política para las audiencias, las tareas de selección y encuadramiento son imprescindibles para mantener vivo el debate democrático (Kinder y Nelson, 2005). Pues, como señalan estos mismos autores, a menudo el problema de los votantes no es tanto el de falta de ideas a la hora de enjuiciar las políticas, como el de tener demasiadas, lo que plantea frecuentes problemas de ambivalencia e inconsistencia, de ahí la necesidad de encuadres que ayuden a integrar información nueva, reducir la disonancia cognitiva, formular opiniones y facilitar su confrontación ${ }^{5}$.

\footnotetext{
${ }^{4}$ Esta diferencia pudo observarse, por ejemplo, en el distinto tratamiento que El País y El Mundo hicieron de la (pre)campaña electoral de 2004, donde el encuadre temático estuvo ilustrado por El País, por contraste con el encuadre más bien episódico de El Mundo.

${ }^{5}$ De acuerdo con Entman, podemos entender la ideología como un "meta-esquema" que sirve para reducir el coste cognitivo de pensar sobre nuevos temas o acontecimientos y para asociarlos con valores e intereses preestablecidos (Entman, 2004: 163).
} 
Ahora bien, las audiencias no son una tabula rasa sobre la cual los medios pueden imponer a discreción una determinada construcción de la realidad. Los estudios sobre la influencia de los medios sugieren, más bien, que la relación entre medios y audiencias es el resultado de una interacción compleja y de una gran diversidad de usos y estrategias por parte de las audiencias. Es de esperar, por tanto, que las audiencias sean receptivas a ciertos encuadres y que rechacen otros, según que resulten congruentes o no con su propia experiencia acumulada y con su cultura política. De ahí la importancia del priming, por cuanto facilita la activación de ideas y sentimientos previamente establecidos, haciendo posible el arraigo de los encuadres. Esto es fundamental para entender el desenlace de las elecciones generales de 2004, pues si la denuncia de manipulación tuvo tanto éxito fue en la medida en que los medios que la promovieron se habían encargado previamente de imprimar la percepción de Aznar en ese mismo sentido.

\section{La agenda de los medios y el conflicto de encuadres}

Como se recordará, la campaña electoral del 14-M fue abruptamente suspendida tras los atentados terroristas del 11-M. ¿Cuál era el clima de opinión antes y después de los atentados? Para responder a la pregunta, hemos hecho un seguimiento de los dos periódicos de más tirada en la capital madrileña: El Mundo y El País. El seguimiento ha consistido en el estudio de sus portadas y editoriales durante el periodo que va del 9 de enero, fecha en que se convocaron oficialmente las elecciones, hasta el mismo día 11-M. Comenzaremos presentando el resultado resumido de dicho seguimiento y, a continuación, expondremos el conflicto de encuadres que se desencadena a partir del 11-M. Terminaremos presentando los diferentes esquemas de voto que surgen a partir del encuadre victorioso (El País).

La muestra recogida a partir del citado seguimiento de prensa correspondiente al periodo de (pre)campaña se compone de 45 titulares de portada y 34 editoriales de El Mundo, así como de 44 titulares de portada y 38 editoriales de El País. No me voy a detener ahora en la presentación de este material, sino que voy tan sólo a subrayar los encuadres característicos de cada uno de estos periódicos a lo largo del periodo de referencia, tal como quedan reflejados en la tabla 3.

En ella tenemos, por un lado, que, desde la perspectiva de El Mundo, la situación política quedaba encuadrada por la contraposición entre un PP presentado como garantía de estabilidad y progreso y un PSOE atrapado en sus propias incoherencias programáticas y en problemas organizativos. El Mundo no rehuyó durante este tiempo el tema de la guerra de Irak, que había sido su principal motivo de discrepancia con Aznar durante la legislatura, pero en un nuevo encuadre que permitía a Aznar desmarcarse de su responsabilidad en la materia y quedar simplemente como "el amigo europeo de la Administración Bush" (editorial 5/02/04). La recomendación de El Mundo no podía ser más clara: voto PP como única garantía de estabilidad y progreso.

Por su parte, El País ponía énfasis en los puntos oscuros de la gestión del PP, haciéndole responsable de los fracasos en materia de inmigración y vivienda, así como 
de la manipulación informativa, representada de manera paradigmática por el engaño de las armas de destrucción masiva que sirvieron de coartada para la guerra de Irak. Un encuadre que parecía diseñado para servir de priming a la gestión de la crisis del 11-M por parte de Aznar, por cuanto fijaba el foco de atención de la audiencia en la credibilidad del gobierno, sobre el trasfondo de manipulación que El País había convertido en marca distintiva del legado de Aznar.

Tabla 3.

Encuadres de El Mundo y El País

(Pre)Campaña.

\begin{tabular}{lcccc}
\hline & \multicolumn{3}{c}{ El Mundo } & \multicolumn{2}{c}{ El País } \\
\cline { 2 - 5 } & PP & PSOE & PP & PSOE \\
\hline Imagen global & $\begin{array}{c}\text { Estabilidad } \\
\text { y progreso }\end{array}$ & $\begin{array}{c}\text { Incoherencia } \\
\text { y desorden }\end{array}$ & $\begin{array}{c}\text { Falta } \\
\text { de credibilidad }\end{array}$ & $\begin{array}{c}\text { Promesa } \\
\text { de consenso }\end{array}$ \\
\hline $\begin{array}{l}\text { Atribución de } \\
\text { responsabilidad }\end{array}$ & $\begin{array}{c}\text { Bonanza económica } \\
\text { y estabilidad política }\end{array}$ & $\begin{array}{c}\text { Crisis del tripartito } \\
\text { catalán }\end{array}$ & $\begin{array}{c}\text { Manipulación } \\
\text { informativa }\end{array}$ & $\begin{array}{c}\text { Exonerado } \\
\text { de responsabilidad }\end{array}$ \\
\hline Evaluación & Positiva & Negativa & Más bien negativa & Más bien positiva \\
\hline
\end{tabular}

Fuente: Elaboración propia a partir de El Mundo y El País (9-01-04 a 11-03-04).

Con estas premisas, la tarea de El País durante la crisis del 11-M era previsible: puesto que la exigencia de responsabilidad a los terroristas era en ese momento poco menos que ilusoria, el encuadre de los atentados por su relación con la guerra de Irak ("en un intento [de Al Qaeda] de extender la guerra de Irak a territorio español", tal como sugería el editorial del día 12) facilitaba una cierta transferencia de responsabilidad al gobierno, cuya colaboración en la guerra de Irak era fácil de precisar, al tiempo que entraba en conflicto frontal con el encuadre del gobierno, según el cual la autoría etarra no dejaba a los votantes indecisos otra salida que el cierre de filas con el gobierno, dada la eficacia mostrada por éste en su lucha contra ETA. Ya no hacía falta hablar de manipulación, toda vez que el priming en ese sentido ya estaba hecho con anterioridad al 11-M. Pero, si los votantes no se habían percatado de ello, el priming de El País sirvió, de paso, a la cadena Ser para deslizar sucesivos indicios que apuntaban en esa misma dirección (la presunta existencia de suicidas en los trenes, así como de un informe del $\mathrm{CNI}$ avalando la pista islámica, etc.; Travesedo de Castilla, 2005).

En cuanto a El Mundo, los atentados del 11-M trajeron de nuevo al centro del escenario el tema que había sido motivo de discrepancia con el gobierno de Aznar, dada su oposición a la colaboración de España en la guerra de Irak. Ante el cariz de los acontecimientos del día 12, cuando las manifestaciones dieron paso a la escenificación del conflicto de 
encuadres (volviéndose contra el gobierno que las había convocado), El Mundo trató de utilizar la jornada de reflexión para apuntalar el encuadre del gobierno sobre la autoría etarra, pero el encuadre se derrumbó ese mismo día con las primeras detenciones de islamistas, tal como recogía la edición del día 14, que reconocía la dificultad que ello comportaba para el gobierno ("Embarazosa situación del Gobierno a pocas horas de la votación").

Tabla 4.

Encuadres de El Mundo y El País del 11-M al 14-M.

\begin{tabular}{|c|c|c|}
\hline & El Mundo & El País \\
\hline Definición del problema & Terrorismo etarra & Terrorismo islamista \\
\hline $\begin{array}{l}\text { Atribución de } \\
\text { responsabilidad }\end{array}$ & ETA & $\begin{array}{c}\text { Guerra de Irak } \\
\text { ¿transferencia de responsabilidad? }\end{array}$ \\
\hline Evaluación del gobierno & $\begin{array}{l}\text { Posible responsabilidad de } \\
\text { Acebes }\end{array}$ & $\begin{array}{l}\text { Gobierno responsable } \\
\text { de manipulación }\end{array}$ \\
\hline
\end{tabular}

Fuente: Elaboración propia a partir de El Mundo y El País.

\section{La experiencia de los entrevistados y los esquemas de voto}

Dedicaremos este último apartado a la experiencia de la crisis por parte de nuestros entrevistados, prestando especial atención a la influencia de los medios. Como ya adelanté, el estudio que propongo aquí no puede hacerse con las encuestas habituales del CIS, sino que está hecho a partir de una investigación ad hoc: el estudio sobre "Voto racional y control democrático: evaluación de la gestión de gobierno y comportamiento electoral. El caso español (1986-2004)"6. Este estudio permitió la realización de 25 entrevistas a trabajadores madrileños de ambos sexos (manuales en el caso de los hombres y no manuales en el de las mujeres) cuya principal característica era la de ser votantes de centro más o menos típicos que, habiendo votado al PSOE con anterioridad (en los años noventa), votaron al PP en las elecciones generales de 2000, cuando este partido obtuvo mayoría absoluta. Estos trabajadores presentan dos pautas de voto principales: por un lado, la de quienes mantuvieron su apoyo al PP (diez casos). Por otro, la de quienes retiraron su apoyo al PP para dárselo al PSOE (nueve casos), a la que podríamos añadir la de quienes, sin haber llegado a votar al PP en 2000 (por haberse abstenido o votado

\footnotetext{
${ }^{6}$ Plan Nacional de Investigación Científica, Desarrollo e Innovación Tecnológica 2000-2003, BSO20000747-C02-01, (IP: Fermín Bouza).
} 
en blanco), votaron al PSOE el 14-M (tres casos). Llamaremos fracción PP a la primera de ellas y fracción PSOE a la segunda?.

Antes de identificar los esquemas de voto de ambas fracciones, conviene tener en cuenta los hábitos de audiencia de los entrevistados antes y después de las elecciones, tal como quedan recogidos en la tabla 5, que recoge también información sobre auto ubicación y distancia ideológica respecto a los principales partidos. Antes de las elecciones, estos votantes se auto ubicaban en el 5,2 de la escala izquierda-derecha y situaban a los principales partidos en el 7,4 (PP), 3,9 (PSOE) y 1,7 (IU). Asimismo, valoraban a Aznar con 6,6, por delante de Zapatero, que tenía una calificación de 6 .

Pues bien, tras las elecciones estos parámetros habían oscilado sensiblemente: su auto ubicación ideológica se había desplazado a la izquierda nada menos que cinco décimas, del 5,2 al 4,7, al tiempo estos votantes habían desplazado al PP nada menos que seis décimas a la derecha, hasta llegar al 8,0. En consonancia, Aznar recibía ahora una calificación de suspenso $(4,8)$, en tanto que Zapatero superaba ahora $(6,7)$ incluso la obtenida por Aznar antes de las elecciones $(6,6)$.

No menos significativos eran los cambios observados en los hábitos de audiencia, pues si bien los niveles de exposición a los medios eran similares antes y después de las elecciones (salvo en el caso de la radio, cuya audiencia se había incrementado notablemente), las preferencias habían cambiado: así, por ejemplo, la preferencia por TVE 1 y Antena 3 entre los informativos de TV había sido sustituida por Tele 5, el canal que más se había desmarcado de la pauta informativa del gobierno durante la crisis. Asimismo, la ligera preferencia por El Mundo frente a El País, como diarios de información general más leídos, se había invertido a favor de este último. Con todo, lo más significativo era la irrupción de la Cadena Ser como la radio más escuchada, lo que parecía un reflejo fidedigno del protagonismo alcanzado por esta emisora durante los días de la crisis.

Con estas premisas, presentaré los esquemas de voto elaborados a partir de la información recogida en las veinticinco entrevistas postelectorales, no sin antes enmarcar brevemente los esquemas de voto en el discurso de los entrevistados sobre los medios y la crisis del 11-M. Para estos entrevistados, percepción política y agenda mediática se solapan hasta confundirse: dado que no tienen una experiencia directa de la política, más allá de su participación electoral, su percepción de la misma es una percepción mediatizada. Así, por ejemplo, cuando se refieren a la relación entre política y corrupción, nuestros entrevistados dan por supuesto que la corrupción está presente en todos los partidos, pero es tarea de los medios determinar su existencia. Por lo tanto, la cuestión no es si hay corrupción, sino si es denunciada por los medios.

\footnotetext{
${ }^{7}$ Huelga decir que las entrevistas en que me apoyo no tienen pretensión alguna de representatividad estadística, sino que su valor heurístico procede de su capacidad para identificar y formular los esquemas de voto que presento más adelante. El mero hecho de realizar más entrevistas no implica necesariamente que dichos esquemas se vean modificados, dada la tendencia a la redundancia discursiva observable en este tipo de estudios.
} 
Tabla 5.

Hábitos de audiencia de los entrevistados

\begin{tabular}{lcc}
\hline & Antes de las elecciones & Después \\
\hline Auto ubicación ideológica (media) & 5,2 & 4,7 \\
Valoración Aznar (media) & 6,6 & 4,8 \\
Valoración Zapatero (media) & 6,0 & 6,7 \\
Informativos TV más frecuentes (n ${ }^{0}$ de casos) & & 5 \\
TVE 1 & 11 & 5 \\
Antena 3 & 9 & 12 \\
Tele 5 & 2 & 1 \\
Telemadrid & 3 & \\
Radio más frecuente (n. ${ }^{0}$ de casos) & & 1 \\
RNE & 3 & 3 \\
COPE & 1 & 1 \\
Onda Cero & 4 & 10 \\
Cadena Ser & 2 & 7 \\
Periódico más frecuente (n. ${ }^{\circ}$ de casos) & & 11 \\
El Mundo & 10 & 2 \\
El País & 8 & 3 \\
La Razón & 2 & 2 \\
Otros & 2 & \\
\hline
\end{tabular}

Fuente: "Voto racional y control democrático".

Empero, la relación de nuestros entrevistados con los medios es ambivalente, toda vez que los necesitan para mantenerse informados, pero no llegan a confiar en ellos plenamente, debido a sus alineamientos políticos e ideológicos. Para nuestros entrevistados, es claro que los medios no se conforman con informar ni con arbitrar el debate político, sino que son parte interesada en la lucha política, como lo prueba la campaña electoral. De ahí que, a la hora de hacer el relato de la crisis, hagan referencia, por lo general, a una diversidad de fuentes (radios, televisiones, etc.). No obstante, la radio, y en particular la Cadena Ser, fue la protagonista mediática de la crisis, incluso entre los votantes del PP, que son los que en mayor medida desconfían del conflicto de encuadres que se desarrolla a partir de las manifestaciones del viernes y que se radicaliza con las detenciones de islamistas del sábado, convirtiendo la jornada de reflexión en una batalla mediática sin precedentes.

La escalada de la tensión culmina, como se recordará, con la aparición de un vídeo de Al Qaeda reivindicando el atentado en la madrugada del domingo electoral, que no hace sino exacerbar el estado de ansiedad y temor de la opinión pública. Entre los votantes PP, cabe la posibilidad de que el gobierno no administrara bien la información, lo que puede 
interpretarse como interés en retrasarla o como simple prudencia, pero lo que está fuera de duda es que los grupos mediáticos de oposición al gobierno estaban interesados en sacar conclusiones sesgadas de ello. Desde la perspectiva de esta fracción de votantes, la victoria socialista se explica como resultado de una transferencia de responsabilidad de los atentados del 11-M a la guerra de Irak, atribución de responsabilidad que estos votantes rechazan enérgicamente y que sólo se explica por la ansiedad del momento y por la presión de los medios.

Para los votantes PSOE, el esquema de justificación del voto pasa por dos momentos: en un primer momento, el relato de los hechos parece confirmar la eficacia del mecanismo de atribución de responsabilidad denunciado por la fracción PP, si bien queda matizado, en un segundo momento, mediante la apelación a la manipulación informativa, consagrando así la victoria del encuadre de El País y la Cadena Ser. Es verdad que al principio hubo resistencia a aceptar un encuadre alternativo al del gobierno incluso entre los propios votantes PSOE, pero una vez puesto en marcha el encuadre de El País y la Cadena Ser, las detenciones del sábado y la aparición del vídeo reivindicativo no hicieron más que corroborar, a los ojos de esta fracción, la sospecha de que el gobierno estaba culminando una larga trayectoria de arbitrariedad informativa que se remontaba, cuando menos, a la huelga general de 2002 y al Prestige, con el agravante de que ahora la opinión pública se enfrentaba al peor episodio conocido de terrorismo ${ }^{8}$.

Finalmente, el gobierno se vio atrapado en un bucle: por un lado, el mecanismo de transferencia de responsabilidad desde los atentados hacia la guerra de Irak remitía a un tema de manipulación sobre las armas de destrucción masiva, tal como El País se había encargado de recordar durante la precampaña. Por el otro, las contradicciones entre el encuadre oficial sobre la autoría etarra de los atentados y las detenciones del sábado alimentaban, a su vez, la atribución de responsabilidad por manipulación y, más concretamente, por uso partidario de la lucha antiterrorista, otro de los temas recurrentes de El País. En último término, todos los argumentos conducian a consagrar la manipulación como argumento recurrente de justificación del voto, tanto más demoledor para el gobierno por cuanto puso en marcha un mecanismo de reevaluación retrospectiva de toda su gestión, de tal suerte que el estímulo inicialmente dirigido a desmontar el encuadre del gobierno sobre la autoría etarra se fue generalizando a las demás parcelas de la actuación gubernamental.

\footnotetext{
${ }^{8}$ El tratamiento informativo de la huelga general de 2002 y del naufragio del Prestige, un petrolero que se hundió cerca de Galicia provocando un desastre ecológico en 2003, fue muy criticado por la opinión pública, llegando a saldarse en el primero de los casos con una sentencia condenatoria de la Audiencia Nacional, que obligó a TVE, controlada por el gobierno, a rectificar
} 
Tabla 6.

Esquemas de voto

\begin{tabular}{|c|c|c|}
\hline & Votantes PP & Votantes PSOE \\
\hline Objeto de evaluación & $\begin{array}{l}\text { La crisis como conflicto } \\
\text { de encuadres }\end{array}$ & La gestión de la crisis \\
\hline Atribución de responsabilidad & $\begin{array}{l}\text { Rechazo de la transferencia de } \\
\text { responsabilidad a la guerra de Irak }\end{array}$ & $\begin{array}{c}\text { El problema no fue tanto la } \\
\text { guerra como la manipulación } \\
\text { informativa }\end{array}$ \\
\hline Explicación & $\begin{array}{l}\text { Se explica por el miedo } \\
\text { (pero no se justifica) }\end{array}$ & $\begin{array}{l}\text { Se explica por inercia } \\
\text { (no era la primera vez) }\end{array}$ \\
\hline Implicación & $\begin{array}{l}\text { El PSOE depende de sus } \\
\text { apoyos mediáticos }\end{array}$ & $\begin{array}{l}\text { Reevaluación retrospectiva } \\
\text { de la gestión del PP }\end{array}$ \\
\hline
\end{tabular}

Fuente: "Voto racional y control democrático".

\section{AMODO DE CONCLUSIÓN: UN MODELO DE VOTO INTEGRADO SUSCEPTIBLE DE CONTRASTACIÓN EMPÍRICA}

He tratado de explicar con ayuda de estos tres modelos los rasgos que me parecen distintivos de cada una de las elecciones generales celebradas en España en la última década: el peso de los factores estructurales, la capacidad de evaluación racional de las políticas públicas y la influencia de los medios de comunicación. Asimismo, he intentado dar soporte empírico a tres hipótesis que me parecen imprescindibles para entender la dinámica electoral de España en los últimos tiempos:

- Desde una perspectiva estructural, la clase ha sido un factor de estabilidad en la evolución electoral, en tanto que la edad ha sido un factor de cambio asociado al ciclo político-electoral. Ahora bien, estas variables no influyen directamente sobre la decisión de voto, sino a través de otras, de ahí su carácter de "variables de control" (véase la parte superior de la figura 1).

- Desde una perspectiva micro, la capacidad de evaluación racional de las políticas ha sido crucial para entender algunos de los acontecimientos electorales de la última década, típicamente las elecciones de 2000, debido a la desideologización del segmento de votantes que ocupa el centro del espectro político-ideológico (véase la parte inferior de la figura).

- Por último, he intentado ilustrar la influencia creciente de los medios, la cual constituye la variable explicativa central del modelo integrado que propongo (véase la parte central de la figura). La conclusión última, tal como se desprende del análisis de las elecciones de 2004, se puede descomponer en dos proposiciones: 
a) Los medios no operan como si las audiencias fuesen meros receptáculos de sus mensajes, sino que las audiencias tienen un papel activo en la selección de aquellos mensajes que son coherentes con su experiencia personal y con su patrón cultural;

b) La eficacia de los medios no radica tanto en su capacidad para imponer su agenda temática como en su capacidad para suministrar los mecanismos de atribución de responsabilidad en virtud de los cuales los votantes evalúan las políticas públicas.

Tales son las premisas analíticas que fundamentan el modelo integrado de voto con el que concluye este artículo. Su capacidad heurística queda a expensas de su contrastación empírica en sucesivas convocatorias electorales, tanto más cuanto la democracia española evolucione en la línea apuntada al principio: progresivamente centrada en los medios.

Figura 1.

Modelo integrado de decisión de voto

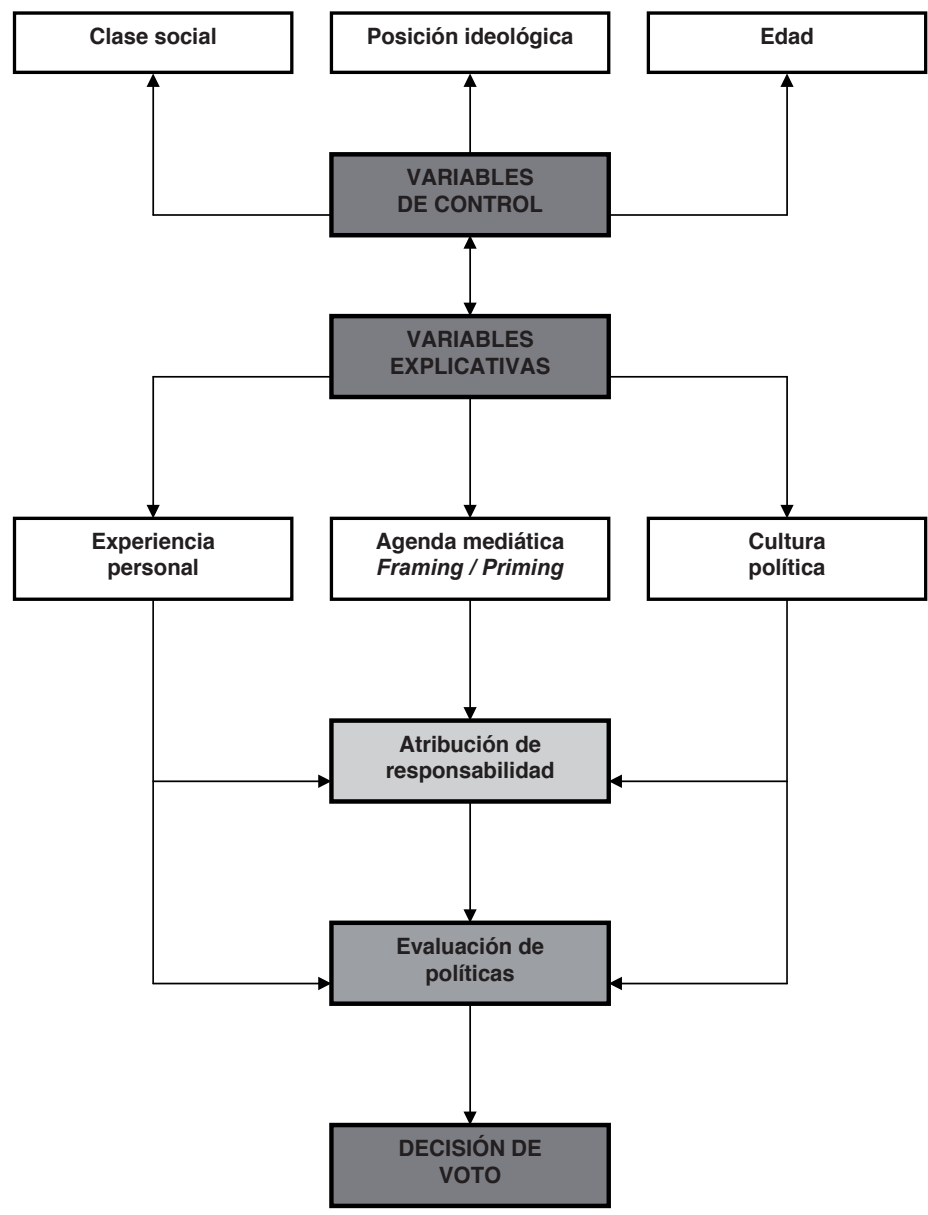




\section{REFERENCIAS BibLIOGRÁFICAS}

Caínzos, M. (2001), "La evolución del voto clasista en España, 1986-2000”, Zona Abierta 96/97: 91-172.

Castells, M. (2003), La era de la información. El poder de la identidad, Madrid, Alianza Editorial.

Entman, R. (1993), "Framing: toward clarification of a fractured paradigm", Journal of Communication, 43: $51-58$.

(2004), Projections of Power. Framing News, Public Opinion, and US Foreign Policy, Chicago, The University of Chicago Press.

Esping-Andersen, G. (1985), Politics against Markets: The Social-Democratic Road to Power, Princeton, Princeton University Press.

(1990), The Three Worlds of Welfare Capitalism, Oxford: Polity Press [Los tres mundos del Estado de Bienestar, Valencia, Alfons el Magnánim, 1993].

Fiorina, M. (1981), Restrospective Voting in American National Elections, New Haven: Yale University Press.

Fraile, M. (2005), Cuando la economía entra en las urnas. El voto económico en España (1979-1996), CIS, Monografías 217.

Giner, S. y L. Moreno (1990), "Centro y periferia: la dimensión étnica de la sociedad española”, en Giner: España. Sociedad y política, Madrid, Espasa-Calpe, pp. 169-198.

González, J. J. (1992), Clases sociales: estudio comparativo de España y la Comunidad de Madrid 1991, Madrid, Consejería de Economía de la Comunidad de Madrid.

(1996), "Clases, ciudadanos y clases de ciudadanos. El ciclo electoral del pos-socialismo (1986-1994)", REIS, 74: 45-76.

(1998), "Política y demoscopia. Los sondeos y las elecciones generales de 1996", Empiria, 1: 181-199.

(2002), "Las elecciones generales de 2000. Voto ideológico/voto racional", Revista Internacional de Sociología, 32: 7-33.

(2004), "Voto y control democrático. Las elecciones del 14-M", CPA Estudios 8, publicación on line del Dpto. de Ciencia Política y de la Administración de la UNED: http://www.uned.es/dcpa/estudios.html.

(2005), "Bases sociales de la política española", en González y Requena: Tres décadas de cambio social en España, Madrid, Alianza Editorial, pp. 253-276.

Hallin, D. y P. Mancini (2004), Comparing Media Systems. Three Models of Media and Politics, Cambridge, Cambridge University Press.

Iyengar, SH. (1991), Is Anyone Responsible? How Television Frames Political Issues, Chicago: The University of Chicago Press. 
Kinder, D. y Th. Nelson (2005), "Democratic Debate and Real Opinions", en Callaghan y Schnell: Framing American Politics, University of Pittsburg Press.

Lewis-Beck, M. S. y M. Paldam (2000), "Economic voting: an introduction", Electoral Studies, 19: 113-121.

Lipset, S. M. et al. (1991), "Are Classes Dying?", International Sociology, 6: 397-410.

(1993), "The Declining Significance of Social Class", International Sociology, 8: 293-316.

Lipset, S. M. y S. Rokkan (1967 [1992]), "Estructuras de division, sistema de partidos y alineamientos electorales", en Batlle, Albert (ed.), Diez textos básicos de ciencia política, Barcelona, Ariel, pp. 231-273.

Manin, B. (1998), Los principios del gobierno representativo, Madrid: Alianza Editorial.

Maravall, J. M. (2003), El control de los políticos, Madrid: Taurus.

McCombs, M. (2004), Setting the Agenda, Oxford: Polity.

Meyer, TH. (2002), Media Democracy. How the Media Colonize Politics, Oxford: Polity.

Olmeda, J. A. (2005), "Miedo o engaño: el encuadramiento de los atentados terroristas del 11-M en Madrid y la rendición de cuentas electoral", Real Instituto Elcano, Documento de Trabajo 24/2005.

Przeworsky, A. (1985 [1988]), Capitalismo y socialdemocracia, Madrid: Alianza Universidad.

Przeworsky, A. y J. Sprague (1986), Paper Stones. A History of Electoral Socialism, Chicago: The University of Chicago Press.

Sampedro, V. (2005), 13-M. Multitudes on line, Madrid: Los Libros de la Catarata.

Swanson, D. (1995), "El campo de comunicación política. La democracia centrada en los medios", en Muñoz Alonso y Rospir (editores), Comunicación Política, Madrid, editorial Universitas, pp 3-24.

Travesedo de Castilla, C. (2005), "Las credibilidades en duelo durante la crisis del 11 al 14-M. La necesidad de desmitificar al grupo Prisa", Razón y Palabra, 47: http://www.cem.itesm.mx/dacs/publicaciones/logos/ anteriores/n47/ctravesedo.html.

Verón, E. (1998), "Interfaces. Sobre la democracia audiovisual evolucionada", en Ferry et al.: El nuevo espacio público, Barcelona, Gedisa. 